$16^{\text {th }}$ International Congress of Metrology, 17002 (2013)

DOI: $10.1051 /$ metrology/201317002

(c) Owned by the authors, published by EDP Sciences, 2013

\title{
The Time and Frequency Measurement Standards of Ukraine
}

Pavel Neyezhmakov, PhD in Technical Science ${ }^{1, a}$, Aleksandr Prokopov, Doctor in Physics and Mathematics ${ }^{1}$, and Vladymir Romanko, PhD in Technical Science ${ }^{1}$.

${ }^{1}$ National Scientific Centre «Institute of Metrology», 61002, Mironositskaya str, 42, Kharkov, Ukraine

\begin{abstract}
The problems of maintenance of the primary measurement standard of the units of time and frequency and national scale of coordinated time of Ukraine (UTC (UA)) are considered, set of measurement standards, its metrological characteristics, comparing results of national scale of the coordinated time of Ukraine with international time scale and time scales of other countries standards are described, participation in forming the international scale of atomic and coordinated time is highlighted. The complex and peculiarities of realization the secondary measurement standards of Ukraine are considered.
\end{abstract}

\section{Historical background of creation of measurement standards of the units of time and frequency in Ukraine}

The first in USSR primary measurement standard of the units of time and frequency was created in National Scientific Center "Institute of Metrology" (NSC IM), Kharkov, Ukraine in 1950.

A group of high-accuracy generators was the basis of it. At that time the measurement standard had the same metrological parameters as national measurement standards of Germany, the USA and Great Britain. In consequence of its accuracy, the measurement standard was one of few others which discovered irregularities in the Erath rotation.

In 1958 for the first time in the USSR an ammonia molecular generator was included into the standard. It laid the beginning to transition of the USSR to reproduction of the units of time and frequency using quantum generators. The uncertainty of the frequency realization using molecular generator was $1 \cdot 10^{-9}$, and that was really a great achievement of national metrology. The research materials on molecular generator were presented to CCTF.

The NSC IM performed foreground research on creation of rubidium and hydrogen standards of frequency, creation of cesium frequency reference and molecular generator on ammonia isotope $\mathrm{N}_{15} \mathrm{H}_{3}$, performed research on creation optical quantum generators, creation of high-accuracy systems for purpose of the frequency comparison.

Since Ukraine has become an independent state, the question on creation own National time and frequency Service and national primary measurement standard was risen again.
To solve important issues of provision the unity of time and frequency measurements Cabinet of Ministers of Ukraine made Decisions to establish State Service of Universal Time and Standard Frequencies (SSTF). The core works at SSTF and their coordination is performed by Ukrainian Metrological Center (UMC) of the SSTF established in the NSC IM, Kharkov.

The technical base of SSTF is the national primary measurement standard of the unit of time and frequency DETU 07-01-97. The general view of one of the premises of the standard is shown at Fig.1.

Figure 1. Equipment of measurement standard of the unit of time and frequency

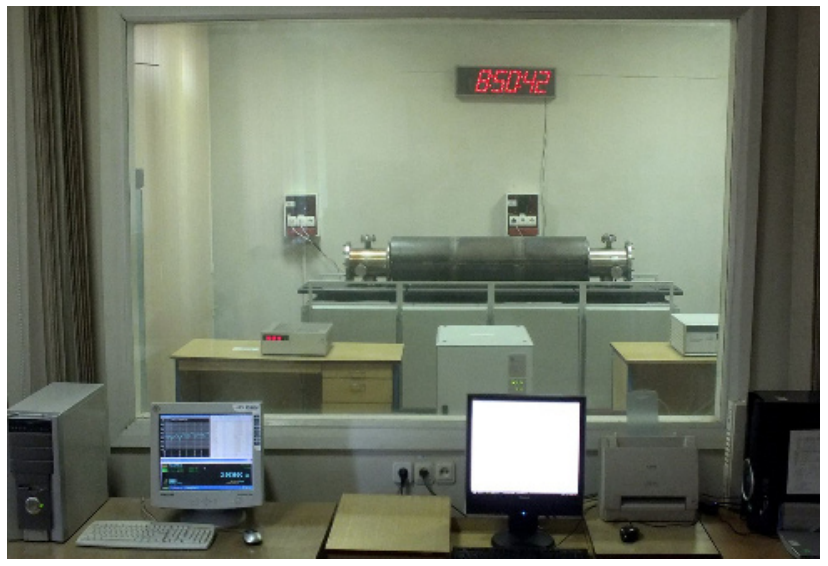

\section{Primary measurement standard of the units of time and frequency of Ukraine DETU 07-01-97}

Functional scheme of measurement standard is shown at Fig.2. At the present time DETU 07-01-97 includes:

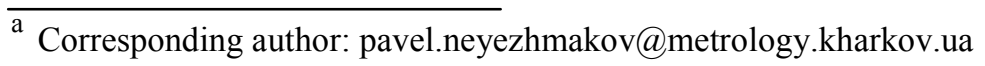


- Facilities for realization and conservation of the unit of time and frequency that includes active hydrogen standard of time and frequency of iMaser 3000 (Hydrogen Maser 1) type, active hydrogen standards of time and frequency of CH1-80 (Hydrogen Masers 2 and 3) type, and cesium standard of time and frequency of 5071 type with improved by metrological characteristics with atomic-beam tube (Cesium Clock 4);

- Automated system of internal comparisons that includes managed via software commutators of signals, phase and frequency comparators, frequency and time interval-meter of GT200-10 type, frequency and time interval-meter of CNT-91 and PC;

- Automated system of external comparisons, based on TTS-2 and TTS-4 systems, that using the signals from Global Navigation Satellite System (GNSS);

- Facilities for formation of the time scale and reference frequencies that includes working time scale formation system, reference frequency formation system, equipment for amplification and multiplication of time and frequency signals;

- Power supply equipment of measurement standard that includes system of the redundant power supplies of standard equipment, the system of thermostatic control of premises (uncertainty of thermostatic control system is no more than $\pm 0,1^{\circ} \mathrm{C}$ ), Automated environment control system (temperature, humidity and atmosphere pressure).

Figure 2. Functional scheme measurement standard of the unit of time and frequency

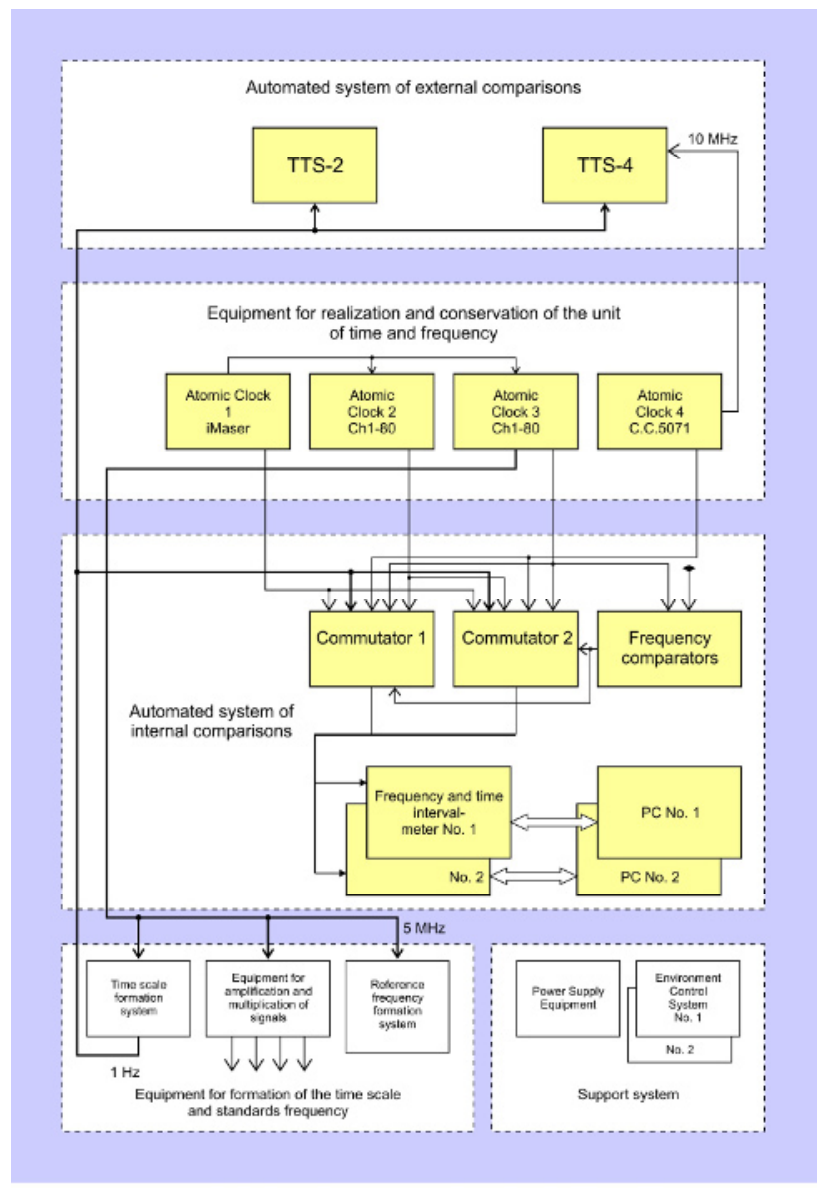

Average annual value of relative frequency difference $\Delta \mathrm{f} / \mathrm{f}$, that characterizes residual bias $\theta_{\mathrm{B}}$ of measurement standard, evaluated with meaning $(4 \ldots .5) \cdot 10^{-15}$ when accepted value is $1 \cdot 10^{-13}$. Root mean square of fractional deviation of 30 -daily evaluations $\Delta \mathrm{f} / \mathrm{f}$ from average annual value for all annual term of observations, which characterizes random uncertainty of realization of units with standard, is $(1,5 \ldots 2,0) \cdot 10^{-14}$ when acceptable value is $5 \cdot 10^{-14}$. Average meaning of group measure frequency is taken as frequency of the measurement standard, that's valid values of all atomic clocks which included to the group team are averaged. Valid values of every atomic clock for 24-ours intervals of measurement time are listed in the monthly information bulletins series E1 and E2 on the web-site of NSC IM [1].

Since 2008 Ukraine participates in forming of International Atomic Time (TAI) and Coordinated Universal Time (UTC). Every month BIPM receives in required format data about time and frequency scales of separate atomic clocks of measurement standard (hydrogen and cesium time and frequency standard UTC(UA)-T(CLOCK). BIPM uses these data together with analogous data of other laboratories to form international scales of atomic TAI and coordinated UTC time.

Table 1 gives values of weight coefficients for time and frequency measurement standards which included to the list of national primary measurement standards of Ukraine and Russian Federation in 2013.

The results of international comparisons of national primary standard of the unit of time and frequency of Ukraine are presented on BIPM web-site (Circular T, CCTF-K001.UTC) the calibration and measuring capabilities (CMC) of Ukraine are present on Key Comparisons Database (KCDB) [2].The total number of the time and frequency CMCs of Ukraine are 30.

\section{Coordinated Universal Time scale of Ukraine}

In 2001 CCTF introduced the concept of time $\operatorname{UTC}(k)$, where index $k$ means determination of one or another laboratory or observatory. In particular, UTC(UA) as maintained by NSC IM, for the present time differs from UTC no more than on 100 ns. This scale is connected with the Atomic Time Scale TA(UA), which was installed in 1996 during metrological certification of national primary measurement standard of the unit of time and frequency and national coordinated time scale UTC(UA).

Installation of UTC(UA) of national primary measurement standard was realized according to validated procedure which based on daily calculation of corrections to working time scale of measurement standard $\mathrm{u}=\mathrm{UTC}(\mathrm{UA})-\mathrm{T}$ (working). Working time scale $\mathrm{T}$ (working) is physically realized as electric signals of pulse form with repetition interval of $1 \mathrm{sec}$. Signals from the working time scale are used for measurements at performance of external comparisons of the standard.

Corrections $u$ are calculated with the help of summarizing of daily variation of the working time scale, which in their turn are determined as a result of evaluation of frequency effective value of the main frequency standard as per data of internal comparisons of group standards. 
Table 1. Weight coefficients of measurement standards of time and frequency of Ukraine and Russia, participated in TAI establishment

\begin{tabular}{|c|c|c|c|c|c|c|c|}
\hline Country & $\begin{array}{c}\text { Measurement } \\
\text { standard code }\end{array}$ & January & February & March & April & $\begin{array}{c}\text { May } \\
\text { Value }\end{array}$ \\
\hline Ukraine & 407881 & 0,11 & 0,118 & 0,111 & 0,113 & 0,139 & 0,1182 \\
\hline Ukraine & 407882 & 0,283 & 0,256 & 0,253 & 0,321 & 0,33 & 0,2886 \\
\hline Ukraine & 407854 & 0,09 & 0,084 & 0,082 & 0,081 & 0,058 & 0,079 \\
\hline Ukraine & 352465 & 0,092 & 0,069 & 0,067 & 0,067 & 0,049 & 0,0688 \\
\hline Russia & 403815 & 0,025 & 0,025 & 0,025 & 0,026 & 0,028 & 0,0258 \\
\hline Russia & 403816 & 0,026 & 0,024 & 0,023 & 0,024 & 0,026 & 0,0246 \\
\hline Russia & 403810 & 0,492 & 0,415 & 0,333 & 0,303 & 0,311 & 0,3708 \\
\hline Russia & 403814 & 0,014 & 0,014 & 0,014 & 0,014 & 0,014 & 0,014 \\
\hline Russia & 403812 & 0,282 & 0,255 & 0,214 & 0,191 & 0,178 & 0,224 \\
\hline Russia & 403809 & $* * * *$ & 0 & 0 & 0 & 0 & 0 \\
\hline
\end{tabular}

Correction $u_{k}$ to the working time scale relatively to the coordinated time scale is determined via equation:

$$
u_{k}=u_{0}+\sum_{k=1}^{K} g_{k}+\sum_{l=1}^{L} a_{l}+\sum_{m=1}^{M} b_{m},
$$

where $g_{k}=-\tau F_{\text {main } g r, k}$ - average variation of the working clock (main custodian) on $k$ interval, which length is $\tau$,

$F_{\text {main }} g r, k$ average value relatively to frequency deviation of the main custodian from the standard frequency on $k$ interval;

$u_{0}-$ correction to the working scale at the initial time $t_{0}(k=0)$;

$K=\left(t_{k}-t_{0}\right) / \tau$ - number of observations intervals;

$a_{l}$ - random failures of the working clock that happened during the interval $t_{k}-t_{0}$;

$L$ - number of failures;

$b_{m}$ - value of correction introduced at correction of the working time scale;

$M$ - number of corrections of the working time scale.

For accounting the corrections to time working scale value $\tau=24$ hours is used.

Information about time scales of the state primary standard in the form of daily values of difference of time scales UTC(UA) and TA(UA) is given in monthly reports series $\mathrm{T}$, which are given on web-site of NSC IM [1]. In 2012 the maximum deviation of the national time scale UTC(UA) from UTC was within limits from -46 $\mathrm{ns}$ to $+38 \mathrm{~ns}$, at allowable deviation of $\pm 100 \mathrm{~ns}$. Average variation of the UTC(UA) in 2012 was $-0,11$ ns per day.

During last 10 years constant comparison of the UTC(UA) with the UTC is performed with application of signals of Global Positioning System (GPS). The main system of external comparisons till August, 2012, was automated system of measurements TTS- 2 based on receiver Motorola Oncore. Beginning from August, 2012, the main system of external comparisons is the automated system of measurements TTS-4 based on receiver Javad TRE-G3T. With the help of system TTS-4 observations in mode "all-in-view" are performed in accordance with the requirements of Technical Directives CCTF of BIPM.

At processing of measurements data average values of difference of time scales T(working)-T(GPS) are calculated during session with the duration of $13 \mathrm{~min}$ (780 measurements), and also average value of this reading for every 24 hours.

At following processing of the measurement results current corrections for the working time scale of the standard UTC(UA)-T(working) and also signal delays in cables are considered. Thereby the average value per each day of the difference of scale of coordinated time of national measurement standard UTC(UA) and time scale T(GPS) of GPS system can be determined. These data are initial for the further comparison of time scales of measurement standards.

The data of measurements by systems TTS- 2 and TTS-4 in GGTTS data format are daily sent to BIPM FTP-servers.

At Fig. 3 the results of comparing the UTC(UA) and UTC during first half of 2013 are given. At Fig. 4 the results of comparing the UTC(UA) with time scale of Germany UTC(PTB) during 2012 are given.

Figure 3. Time scale difference UTC(UA) and UTC

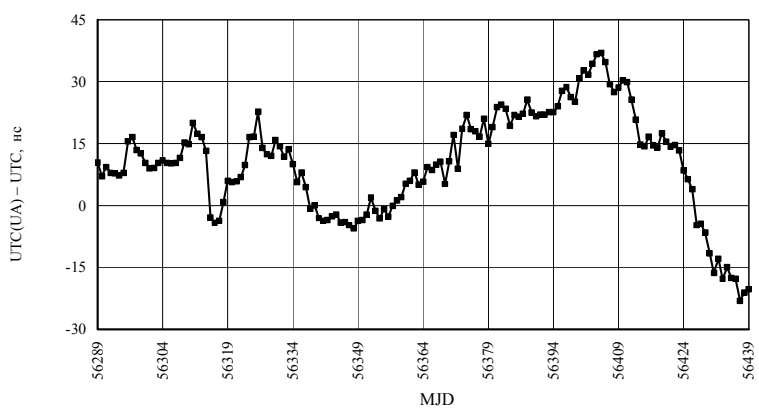

Figure 4. Time scale difference UTC (UA) and UTC(PTB)

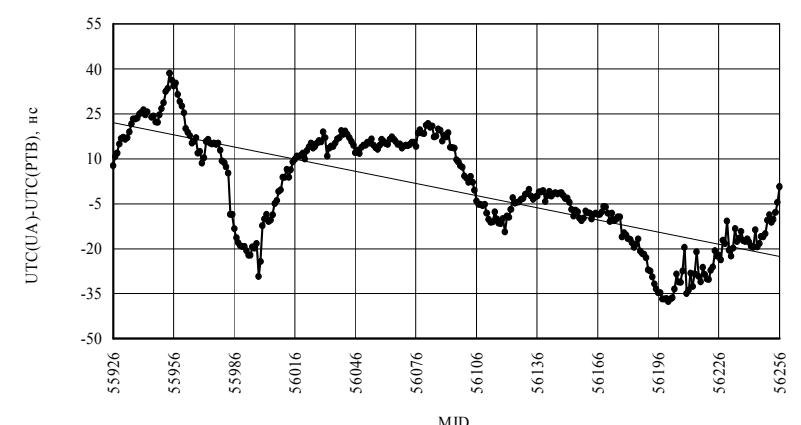




\section{Secondary measurement standard VETU 07-01-03-10 of SE "Ukrmetrteststandard", Kiev}

VETU 07-01-03-10 includes three hydrogen $(\mathrm{H})$ maser standards, equipment for internal and external comparisons. Relative standard measurement uncertainty of secondary standard does not exceed value $1,5 \cdot 10^{-13}$. Scale differences between UTC (KI) of VETU 07-01-0310 and UTC (UA) are not exceed 1 mcs. Comparisons of the secondary measurement standard with the DETU 0701-97 are performed every 3 months.

\section{Secondary measurement standard VETU 07-01-05-11 of NSC IM, Kharkov}

Three $\mathrm{Rb}$ frequency standards are the basis of Secondary time and frequency measurement standard: two rubidium $(\mathrm{Rb})$ frequency standards based on rubidium modules of FE5680A type and also Rb frequency standard based on typical time and frequency standard in order to ensure its external control by output frequency. Relative standard measurement uncertainty of secondary standard is estimated as value $5,2 \cdot 10^{-14}$.

Automatic correction of frequency of Rb-standards based on GPS system is realized. The microcontrolling module of control and monitoring receives the signal from GPS-receiver with the frequency of $10 \mathrm{MHz}$, synchronized as per phase with UTC time. At the same time the control module receives the signal from rubidium module FE5680A. Constant measurement of deviation of the module output frequency relatively to the receiver frequency is performed in the module with the help of phase comparator performed on a specialized microchip and, if necessary, commands on its agility as per frequency are given as per Protocol RS-232.

\section{The results of the comparison of secondary measurement standards with DETU 07-01-97}

Monitoring of deviation of the time scale UTC(KI) of the secondary standard VETU 07-01-03-10 off UTC(UA) is performed with the help of receivers of signals of global navigation satellite systems (GNSS). Monitoring of deviation of the time scale $\mathrm{UTC}(\mathrm{KH})$ of VETU 07-01-05-11 off UTC (UA) is performed via direct measurements with the help of internal comparisons system DETU 07-01-97. The results of monitoring are given in Fig. 5.
Figure 5. Difference of the time scale of secondary standards from the time scale of the primary standard

ns

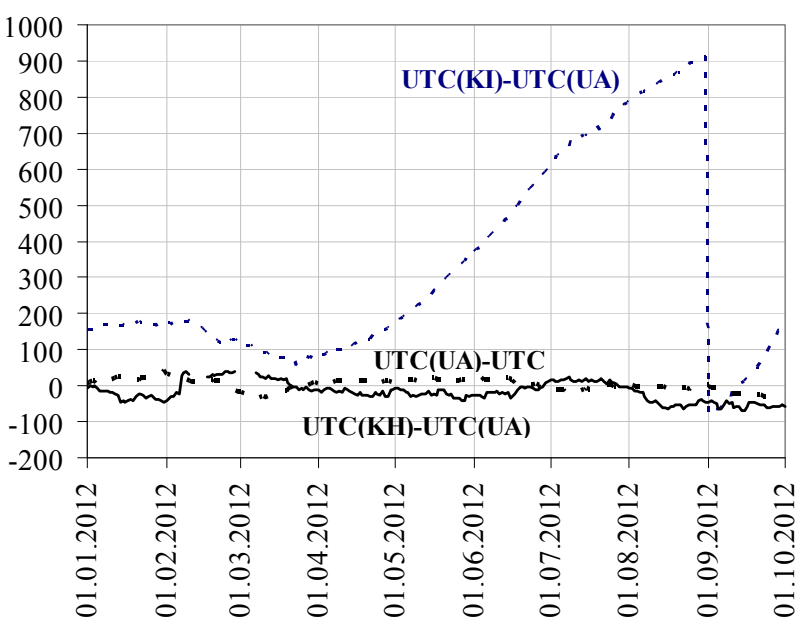

It is obvious from the monitoring results that beginning from April, 2012, the secondary standard of the State Enterprise "Ukrmetrteststandard" has constant time scale variation UTC(KI) relatively to UTC (UA) within 100-200 nc per month. Correction of the time scale of the standard for minus 1000 ns was made on 01.09.2012. The difference of the time scale UTC(KH) of the secondary standard VETU 07-01-05-11 and UTC (UA) in 2012 did not exceed -68 ns (see Pic. 5) systematic variation almost does not exist, that is provided by presence of automatic frequency adjustment system of Rb-standards as per signals of GNSS in the secondary standard VETU 07-01-05-11.

\section{References}

1. www.metrology.kharkov.ua

2. http://kcdb.bipm.org 